\title{
Combining the Classical and Lumped Diesel Particulate Filter Models
}

\author{
Christopher Depcik \\ Univ. of Kansas
}

\begin{abstract}
The growing presence of Spark Ignition Direct Injection (SIDI) engines along with the prevalence of direct injected Compression Ignition (CI) engines results in the requirement of Particulate Matter (PM) exhaust abatement. This occurs through the implementation of Gasoline Particulate Filters (GPFs) and Diesel Particulate Filters (DPFs). Modeling of GPFs and DPFs are analogous because of the similar flow patterns and wall flow PM capture methodology. Conventional modeling techniques include a two-channel (inlet/outlet) formulation that is applicable up to three-dimensions. However, the numerical stiffness that results from the need to couple the solution of these channels in compressible flow can result in relatively long run times. Previously, the author presented a lumped DPF model using dynamically incompressible flow intended for an Engine Control Unit (ECU) in order to generate a model that runs faster than real time using a high-level programming language. Building on the favorable outcomes of temperature evolution from this prior effort, this work enhances the model to predict compressible flow gas dynamics in order to match the evolution of pressure drop. Another enhancement is the inclusion of deep bed filtration within the wall, and the transition to the cake layer. Results show comparable temperature profiles with the dynamically incompressible model with a pressure drop that follows appropriately by linking through the ideal gas model. However, solving chemical species as an independent equation separate from compressible flow still deviates significantly from the classical two-channel approach.
\end{abstract}

CITATION: Depcik, C., "Combining the Classical and Lumped Diesel Particulate Filter Models," SAE Int. J. Engines 8(3):2015, doi:10.4271/2015-01-1049.

\section{INTRODUCTION}

The control of PM that emanates from SIDI and CI engines is of significant concern for the automotive industry. This is because PM can infiltrate the alveoli in humans and transport toxic chemicals absorbed on the surface of the particle, subsequently leading to lung related illnesses [1]. As a result, the automotive industry now widely implements PM filtration in the exhaust. In particular, DPFs are used for CI engine exhaust with GPFs currently being considered for SIDI engines.

The operation of a DPF (or analogous GPF) includes the storage of $\mathrm{PM}$ in and on top of a porous wall with its eventual regeneration in an oxidative environment. This can be accomplished actively utilizing $\mathrm{O}_{2}$ or passively by employing $\mathrm{NO}_{2}$; often through synchronization with an upstream Oxidation Catalyst. This need to modulate device operation has led to the development of simulation tools in order to reduce the number of experiments needed by predicting the pressure drop through the device and its temperature evolution. Broadly speaking, the models typically fall into one of two categories; those designated to operate within an Engine Control Unit (ECU) [2] and the classical two-channel approach []ㅡ.

The ECU approach is designed for computational speed and is still often based on the two-channel model originally posed by Bissett [4]. In a previous effort by the author, the research in this area was reviewed and a derived model was generated that used dynamically incompressible flow to simulate the flow through the device while creating lumped zones in order to predict the temperature evolution. Utilizing a high-level coding language that runs significantly slower than traditional programming languages [ $[5]$, the filter temperature evolution could be predicted relatively accurately in a faster than real time manner. This was because only one term in the governing energy equations was influenced by compressible flow. However, decoupling temperature and pressure by using dynamically incompressible flow (effectively negating the ideal gas law), the model was unable to simulate the pressure drop during warm-up and PM regeneration experiments.

As a result, this effort seeks to merge the two models by simulating temperature using the ECU approach while estimating the pressure drop using the two-channel methodology. In specific, the temperature profile through the device is generated by the ECU version that is then utilized as a constant within the two-channel model removing the need to solve for the channel temperatures. Moreover, deep bed filtration is included in the model formulation in order to simulate the pressure drop evolution in the device more accurately. Finally, model run times are given in order to document the computational expense of the combined model.

\section{DPF MODEL DERIVATION}

In a previous effort, instead of simulating both the inlet and outlet channels along with a porous wall, model derivation followed a lumped approach through the creation of a variable number of zones 
[2]. The prior efforts found that temperature could be computed in this manner effectively when compared to the classical two-channel approach because only one variable was affected. As a result, this work will employ the lumped approach for temperature while bringing in the classical approach for the pressure drop through the filter. For brevity, a succinct summary of the previous model formulation is presented and only the new variables are defined since the rest of the parameters are available in the previous paper.

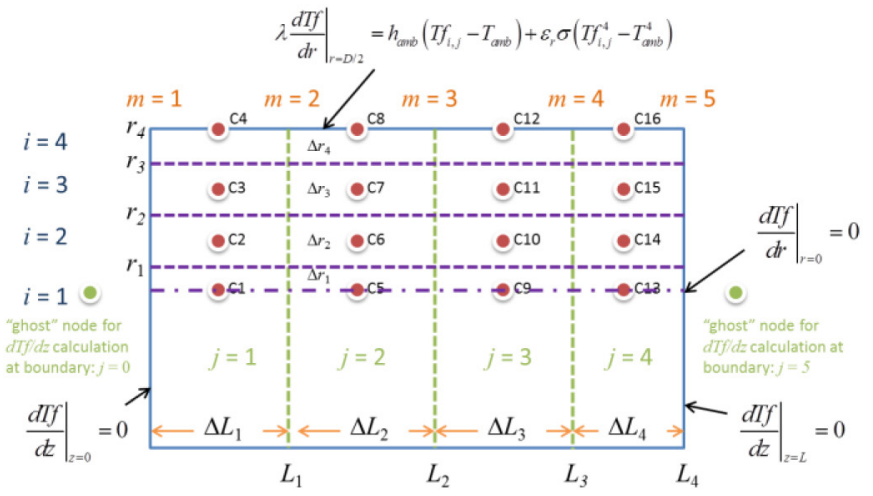

Figure 1. Graphical schematic of the lumped DPF model illustrating pertinent lengths, boundary conditions, and subscript nomenclature.

\section{Filter and Gas Energy Equations}

The energy equation for the filter includes an energy storage term on the left hand side:

$$
\begin{aligned}
\left(\rho_{s} c_{s} V s_{i, j}+\rho_{f} c_{f} V f_{i, j}\right) \frac{d T f_{i, j}}{d t} & = \\
\dot{Q}_{\text {cond,axial }} & +\dot{Q}_{\text {cond }, \text { radial }}+\dot{Q}_{\text {conv }}+\dot{Q}_{\text {wall }}+\dot{Q}_{\text {reac }}
\end{aligned}
$$

with the right hand side adding the influences of axial conduction along the filter $\left(\dot{Q}_{\text {cond,axial }}\right)$, radial conduction in the normal direction $\left(\dot{Q}_{\text {cond,radial }}\right)$, convection between the channel gases and the filter wall $\left(\dot{Q}_{\text {conn }}\right)$, an enthalpy flow through the wall $\left(\dot{Q}_{\text {wall }}\right)$, and the influence of the PM exothermic reactions $\left(\dot{Q}_{\text {reac }}\right)$. Of note, the subscript nomenclature indicates $i$ for the radial direction and $j$ for the axial direction as indicated in Figure 1.

Writing a lumped energy equation for the gas employing an average gas temperature that includes both the inlet and outlet channels equals:

$$
\bar{\rho}_{j} c_{p} V e s_{i, j} \frac{d T_{i, j}}{d t}=\dot{Q}_{i n}+\dot{Q}_{o u t}-\dot{Q}_{c o n v}
$$

The left hand side describes the energy stored by the gas that is often considered negligible. However, keeping this term allows this equation to be solved with other equations as an array of Ordinary Differential Equations (ODEs).

The prior modeling effort employed dynamically incompressible flow; hence, only one value for gas density was utilized throughout the entire DPF in Eqn. (2). In this effort, a single representative inlet and outlet channel will be employed in order to calculate the pressure drop of the DPF using compressible flow. Therefore, in Eqn. (2), the gas density is evaluated as an average of inlet and outlet channels:

$$
\bar{\rho}_{j}=\frac{1}{2}\left[\frac{1}{2}\left(\rho_{I, m}+\rho_{I, m+1}\right)+\frac{1}{2}\left(\rho_{I I, m}+\rho_{I I, m+1}\right)\right]
$$

with the subscript nomenclature of $m$ employed in order to differentiate between values at the boundaries of Figure 1 and those computed in the middle of the zones (i.e., $i \& j$ ). This is required in order to synchronize with the common boundary conditions for the classical two-channel model. Moreover, the subscripts $I$ and $I I$ refer to the inlet and outlet channels, respectively, of the classical model.

Since only one representative set of channels will be simulated, the value of temperature will be a weighted average in the radial direction:

$$
\bar{T}_{j}=\sum_{i} \frac{\dot{m}_{i, j} T_{i, j}}{\dot{m}_{\text {total }}}
$$

However, as just indicated, these values are defined in the middle of the zone. Therefore, they must be translated to the boundary temperatures of each zone:

$$
\begin{array}{cc}
T_{\text {III }, m}=T_{\text {inlet }} & @ z=0 \\
T_{\text {III }, m}=\frac{1}{2}\left(\bar{T}_{j-1}+\bar{T}_{j}\right) & @ 0<z<L_{t}
\end{array}
$$

$$
T_{I I I, m}=\bar{T}_{j} @ z=L_{t}
$$

with the last assumption indicating that the exit temperature change over the half-cell is negligible. Of note, for the temperature exiting the DPF, this is the same boundary condition utilized in the previous effort.

Now, from this information, the temperature derivative between the boundaries can be specified as:

$$
\left.\frac{d T_{I I I}}{d z}\right|_{m}=\frac{\left.T_{I I I}\right|_{m+1}-\left.T_{I I I}\right|_{m}}{\Delta L_{j}}
$$

This will act as a constant within the ODE solver for the channel equations, effectively removing the need to employ the conservation of energy in the solver. In other words, the lumped model will provide the temperature profile through the DPF and the channel equations will simply employ this result. 


\section{PM Mass Equations}

The previous effort only employed a PM mass balance for the cake layer and neglected deep bed filtration. This has been expanded here based on the unit collector model pioneered by Konstandopoulos and Johnson [ㄷ] and later updated by Konstandopoulos et al. $[\underline{7}, \underline{8}, \underline{9}, \underline{10}]$. This effort slightly modifies this model for inclusion in the lumped zone approach.

In the model, there are now equations for both the PM stored in the wall $(\mathrm{mw})$ and cake layer $(\mathrm{ms})$, respectively:

$$
\begin{gathered}
\frac{d\left(m w_{i, j}\right)}{d t}=\Phi_{i, j} \dot{m} s_{i, j}-\chi_{i, j} m w_{i, j} \\
\frac{d\left(m s_{i, j}\right)}{d t}=\left(1-\Phi_{i, j}\right) \dot{m} s_{i, j}-\chi_{i, j} m s_{i, j}
\end{gathered}
$$

where $\Phi$ is a partition coefficient that determines the fraction of mass collected in the first slab that contributes to cake formation. It is calculated using the characteristic dimension of a unit spherical collector ( $d c$ and $d c_{0}$ for the initial value of an unloaded wall):

$$
\Phi_{i, j}=\frac{d c_{i, j}^{2}-d c_{0}^{2}}{(\psi b)^{2}-d c_{0}^{2}}
$$

with $\psi$ a calibration constant and $b$ equal to the unit cell diameter:

$$
b^{3}=\left(1-\varepsilon_{0}\right) d c_{0}^{3}
$$

and $\varepsilon_{0}$ is the porosity of a clean filter. The initial value of the collector diameter of an unloaded wall is calculated as:

$$
d c_{0}=\frac{3}{2}\left(\frac{1-\varepsilon_{0}}{\varepsilon_{0}}\right) d_{\text {pore }}
$$

where $d_{\text {pore }}$ is the pore diameter of the filter. Of note, this is a characteristic dimension of a solid "spherical" collector. The numerator $\left(1-\varepsilon_{0}\right)$ indicates the amount of the pore that is the solid fraction; whereas, the denominator $\left(\varepsilon_{0}\right)$ is the open fraction of the pore.

The current value of the unit spherical collector is based on the amount of deposited particulate in the wall:

$$
\frac{4 \pi}{3}\left(\frac{d c_{i, j}}{2}\right)^{3}=\frac{m w p_{i, j}}{\rho_{s w}}+\frac{4 \pi}{3}\left(\frac{d c_{0}}{2}\right)^{3}
$$

Effectively, this equation determines the diameter of a unit spherical collector (hence, volume as indicated through third power) as a function of the volume of PM per pore and the initial unit spherical collector value. Investigating Eqn. (14), one can see that this equation effectively grows the solid fraction of the pore that is used to capture the PM through the mechanisms of Brownian diffusion and interception.

The volume of PM per pore involves the entire PM that is stored in the wall zone that is currently being analyzed, divided by the total number of pores $(\mathrm{Np})$ in the wall at the current location:

$$
m w p_{i, j}=\frac{m w_{i, j}}{N p_{i, j}}
$$

$$
N p_{i, j}=\frac{V e o_{i, j}}{\frac{4 \pi}{3}\left(d_{\text {pore }} / 2\right)^{3}}
$$

where the denominator is an equivalent pore volume and Veo is the empty volume of the wall at the location under analysis:

$$
V e o_{i, j}=\varepsilon_{0} V f_{i, j}
$$

It is anticipated that $N p$ is grossly overestimated as it assumes that all pores will be available for PM capture, which is untrue. This is remedied through $\rho_{s w}$, the packing density of the PM particles in the filter structure. This is taken as a calibration constant in this effort similar to Konstandopoulos et al. [11] that found values for this parameter between 8.26 and $14.10 \mathrm{~kg} \mathrm{~m}^{-3}$. Of note, the methodology presented in this paper deviates from the conventional unit collector approach that divides the filter wall into slabs and tracks the amount of particulate accumulated in each slab. This version is no longer dependent on a discretization in the wall direction, and instead lumps all PM capture into a single slab. Hence, the value of the packing density will be different from the previous studies indicated.

The mass flow rate to the wall comes from previous efforts of the author $[\underline{12}, \underline{13}]$ :

$$
\dot{m} s_{i, j}=\bar{\rho}_{j} \bar{u}_{s} Y_{d} S_{i, j}
$$

utilizing the average gas density, the mass fraction of PM in the inlet channel gas $\left(Y_{d}\right)$, and the average wall flow velocity to the surface since it does not change significantly in the axial direction:

$$
\bar{u}_{s}=\sum_{m} u_{s, m} / N z
$$

where $N z$ is the number of boundaries and the calculation for $u_{s, m}$ is provided later in this paper. The inlet channel surface area for the particular zone under analysis: 


$$
S_{i, j}=\frac{N c_{i}}{2}\left[4\left(d-2 t s_{i, j}\right)\right] \Delta L_{j}
$$

In Eqns. (9) and (10), PM combustion is calculated as a function of the local oxygen mass fraction:

$$
\chi_{i, j}=\frac{S_{p} \bar{\rho}_{j} Y_{i, j, \mathrm{O}_{2}} k_{\mathrm{O}_{2}} W_{\mathrm{C}_{(s)}}}{\alpha_{\mathrm{O}_{2}} W_{\mathrm{O}_{2}} \rho_{s}}
$$

similar to the previous effort [2] but now factoring the change in density along the DPF.

\section{Channel Equations}

As indicated in the introduction, the purpose of this paper is to merge the classical two-channel model approach with the lumped temperature equation. Therefore, the equations for the inlet and outlet channels in compressible flow must be calculated using the temperature (via Eqns. (5), (6), (7), (8)) and the PM in each zone (Eqn. (9) and (10)). Instead of computing an average PM thickness across the entire DPF as accomplished prior, an average PM thickness is calculated across only the radial zones:

$$
\overline{t s_{j}}=\frac{1}{2}\left[d-\sqrt{d^{2}-\frac{m s t_{j}}{\left(\frac{N c_{t}}{2}\right) \Delta L_{j} \rho_{s}}}\right]
$$

using the total PM stored on the surface in these zones:

$$
m s t_{j}=\sum_{i} m s_{i, j}
$$

In order to calculate the changing pressure drop through the wall with loading, the average PM per pore in the radial direction must be calculated. First, the total amount of PM in the wall is calculated radially:

$$
m w t_{j}=\sum_{i} m w_{i, j}
$$

Similar to Eqn. (15), the average wall PM mass per pore is found:

$$
{\overline{m w p_{j}}}_{j}=\frac{m w t_{j}}{N p t_{j}}
$$

where the total number of pores in the zone is calculated as a fraction of the total number of pores in the filter:

$$
N p t_{j}=\frac{N p a}{L_{t}} \Delta L_{j}
$$

and Npa is the total number of pores in the filter:

$$
N p a=\frac{\varepsilon_{0} \cdot V f t}{\frac{4 \pi}{3}\left(d_{\text {pore }} / 2\right)^{3}}
$$

with $V f t$ equal to the total solid filter volume. Now, from Eqn. (25), Eqn. (14) can be used to find the average unit spherical collector in the zone under study $\left(d c t_{j}\right)$. Then, the porosity shrinks as a function of this unit collector diameter:

$$
\varepsilon_{j}=1-\frac{d c t_{j}^{3}}{d c_{0}^{3}}\left(1-\varepsilon_{0}\right)
$$

By using the Kuwabara unit cell,

$$
f\left(\varepsilon_{j}\right)=\frac{2}{9} \frac{\left[2-\frac{9}{5}\left(1-\varepsilon_{j}\right)^{1 / 3}-\varepsilon_{j}-\frac{1}{5}\left(1-\varepsilon_{j}\right)^{2}\right]}{\left(1-\varepsilon_{j}\right)},
$$

the wall permeability can be found in each zone:

$$
\mathbf{K}_{w, j}=\mathbf{K}_{w 0}\left(\frac{d c t_{j}}{d c_{0}}\right)^{2} \frac{f\left(\varepsilon_{j}\right)}{f\left(\varepsilon_{0}\right)}
$$

where $\mathrm{K}_{w 0}$ is the permeability of the clean filter. Of note, for the PM thickness via Eqn. (22) and the wall permeability of Eqn. (30), these values are held constant across the zone. For example, when $m$ is iterated from 1 to 2 across that zone, $j$ is equal to 1 .

Now, by including a specified PM permeability for the cake layer $\left(\mathrm{K}_{s}\right)$ the velocity in the PM and wall layers can be found for a representative channel in that zone employing Darcy's law and area conservation [12]. In particular, defining a pressure at the interface between the PM and wall layers $\left(p_{m}\right)$ the velocity through the PM and wall layers can be determined:

$$
u_{s, m}=\frac{\left(p_{I, m}-p_{m, m}\right) K_{s}}{\mu \cdot \overline{t s_{j}}}
$$

$$
u_{w, m}=\frac{\left(p_{m, m}-p_{I I, m}\right) K_{w, j}}{\mu \cdot t w}
$$

Employing an assumption that the density in the PM and wall layers is calculated from the ideal gas law and the average of the pressure across that zone [14]: 


$$
\begin{gathered}
\rho_{l, m}=\frac{\frac{1}{2}\left(p_{I, m}+p_{m, m}\right)}{R T_{I I I, m}} \\
\rho_{w, m}=\frac{\frac{1}{2}\left(p_{m, m}+p_{I I, m}\right)}{R T_{I I I, m}}
\end{gathered}
$$

and ensuring conservation of mass (seen via the channel equations via Eqns. (38) and (1ㅡ), presented later)

$$
\frac{\rho_{l} u_{s}}{4\left(d-2 \overline{t s_{j}}\right)}=\frac{\rho_{w} u_{w}}{4 d}
$$

allows one to solve for the pressure at the interface along with the densities and velocities. In particular, Eqns. (31), (32), (33), (34), (35) represent a set of five equations and five unknowns; i.e., $u_{s}, u_{w}, \rho_{s}, \rho_{w}$, and $p_{m}$ with $p_{I}$ and $p_{I I}$ specified (actually calculated via an ODE solver, discussed later in this section).

Now, the representative channel equations can be solved by marching forward in the axial direction. Similar to previous efforts [15], the density and velocity in each channel $(I$ and $I I)$ is combined into an effective flow term:

$$
G=\rho u
$$

Drawing from experience with the two-channel model, a slight adaptation is made here with respect to this variable for the inlet channel. In specific, the velocity in the inlet channel $\left(u_{I}\right)$ is equal to zero at the end of the channel. This has previously caused numerical difficulties for the author when employing the Newton-Raphson iteration scheme (discussed in next section) for determining the initial pressure in the outlet channel since it appears in the denominator. Hence, a variable transformation is made for the inlet channel:

$$
u_{\alpha, m}=u_{I, m}+1
$$

Therefore, $u_{\alpha}$ is equal to one at the end of the channel preventing the possible division by negligible velocity term when solving for the next outlet channel initial pressure.

Moreover, in order to reduce the numerical difficulty in resolving a differential-algebraic set of equations, the derivative of the ideal gas law is employed [15]. As a result, the governing ordinary differential equations for the inlet channel are:

$$
\frac{d G_{\alpha}}{d z}=-\frac{4 \rho_{l} u_{s}}{d-2 \overline{t s_{j}}}+\frac{d \rho_{I}}{d z}
$$

$$
\begin{gathered}
\frac{d p_{I}}{d z}=-\frac{2\left(G_{\alpha}-\rho_{I}\right)}{\rho_{I}}\left[\frac{d G_{\alpha}}{d z}-\frac{d \rho_{I}}{d z}\right]+ \\
\frac{\left(G_{\alpha}-\rho_{I}\right)^{2}}{\rho_{I}^{2}} \frac{d \rho_{I}}{d z}-\frac{\mu F\left(G_{\alpha}-\rho_{I}\right)}{\left(d-2 \overline{t s_{j}}\right)^{2} \rho_{I}} \\
\frac{d \rho_{I}}{d z}=\frac{1}{R T_{I I I}^{2}}\left(T_{I I I} \frac{d p_{I}}{d z}-p_{I} \frac{d T_{I I I}}{d z}\right)
\end{gathered}
$$

And for the outlet channel:

$$
\frac{d G_{I I}}{d z}=\frac{4 \rho_{w} u_{w}}{d}
$$

$$
\begin{gathered}
\frac{d p_{I I}}{d z}=-\frac{2 G_{I I}}{\rho_{I I}} \frac{d G_{I I}}{d z}+\frac{G_{I I}^{2}}{\rho_{I I}^{2}} \frac{d \rho_{I I}}{d z}-\frac{\mu F G_{I I}}{d^{2} \rho_{I I}} \\
\frac{d \rho_{I I}}{d z}=\frac{1}{R T_{I I I}^{2}}\left(T_{I I I} \frac{d p_{I I}}{d z}-p_{I I} \frac{d T_{I I I}}{d z}\right)
\end{gathered}
$$

with the PM, wall density and velocity updated within the ODE solver routine using the computed values of pressure for the inlet and outlet channels. Temperature is included in the ODE solver routine using a constant derivative for each zone as calculated using Eqn. (8) so that it changes dynamically with the pressure and density according to compressible flow.

\section{Species Equation and Boundary Conditions}

Similar to the previous effort, the chemical species equation includes the advection of gas written using an upwind methodology and the reactions happening within the zone:

$$
\frac{d \bar{C}_{i, j, k}}{d t}+\frac{u_{i, j} \bar{C}_{i, j, k}-u_{i, j-1} \bar{C}_{i, j-1, k}}{\frac{1}{2}\left(\Delta L_{j}+\Delta L_{j-1}\right)}=\overline{R R}_{i, j, k}
$$

While it is possible to include the chemical species equations for the inlet and outlet channels along with the flow through the PM and wall layers [13], this will significantly increase the computational expense of the solver slowing down the numerical run time. Moreover, it was desired to understand whether this would be needed in order to simulate chemical species accurately.

The additional boundary conditions needed for this mixed model include the representative channel inlet velocity:

$$
\left.u\right|_{z=0}=\frac{\dot{m}_{\text {total }}}{\left.\rho_{I}\right|_{z=0} \frac{N c_{i}}{2}\left(d-2 \cdot \overline{t s_{j=1}}\right)^{2}}
$$


The velocities at the boundaries:

$$
\begin{aligned}
& \left.u_{\alpha}\right|_{z=L_{t}}=1 \\
& \left.u_{I I}\right|_{z=0}=0
\end{aligned}
$$

The exit pressure of the outlet channel equal to the ambient:

$$
\left.p_{I I}\right|_{z=L_{t}}=p_{a m b}
$$

A Newton-Raphson iteration technique is used along with Eqn. (48) in order to calculate the initial pressure for the inlet and outlet channels. In specific, the change in outlet pressure for each iteration is calculated based on the prior two guesses and the latest value for the velocity at the exit of the inlet channel:

$$
\left.d p_{I I}\right|_{z=0}=\frac{\left.p_{I I}^{\prime}\right|_{z=0}-\left.p_{I I}\right|_{z=0}}{\left(1-\left.u_{\alpha}\right|_{z=L_{l}}\right) /\left(\left.u_{\alpha}^{\prime}\right|_{z=L_{t}}-\left.u_{\alpha}\right|_{z=L_{t}}\right)}
$$

where the prime indicates the latest value employed. Hence, a new pressure for the initial outlet pressure is calculated:

$$
\left.p_{I I}^{\prime \prime}\right|_{z=0}=\left.p_{I I}^{\prime}\right|_{z=0}+\left.d p_{I I}\right|_{z=0}
$$

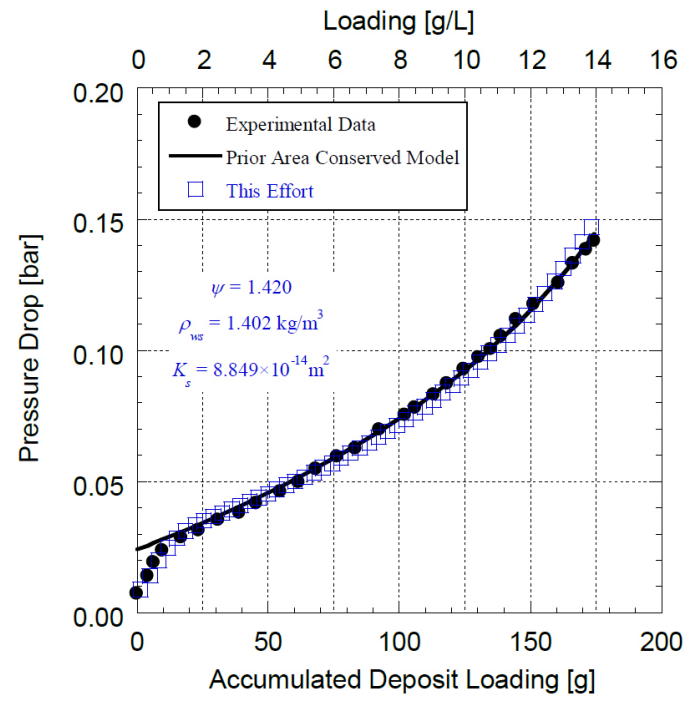

Figure 2. Comparison between prior area conserved model that simulated only the cake layer and the deep bed filtration model presented in this effort along with experimental data [16].

In order to ensure consistency with the exit ambient pressure via Eqn. (48), the initial pressures for the inlet and outlet channels are adjusted accordingly:

$$
\left.p_{I I}\right|_{z=0}=\left(\left.p_{I I}^{\prime \prime}\right|_{z=0}-\left.p_{I I}^{\prime}\right|_{z=L_{l}}\right)+p_{a m b}
$$

$$
\left.p_{I}\right|_{z=0}=\left(\left.p_{I}^{\prime}\right|_{z=0}-\left.p_{I I}^{\prime}\right|_{z=L_{t}}\right)+p_{a m b}
$$

\section{RESULTS AND DISCUSSION}

For a computing platform, Matlab v7.12 (R2011a) was utilized because of its ability to post-process the results as they were generated. This decreases development time by speeding the debugging process through immediate evaluation of the simulation outcomes []. In addition, Matlab includes embedded ODE solvers (ode15s used in this effort) that can be employed in order to solve Eqns. (1), (2), (9), (10), and (38), (39), (40), (41), (42), (43), (44).

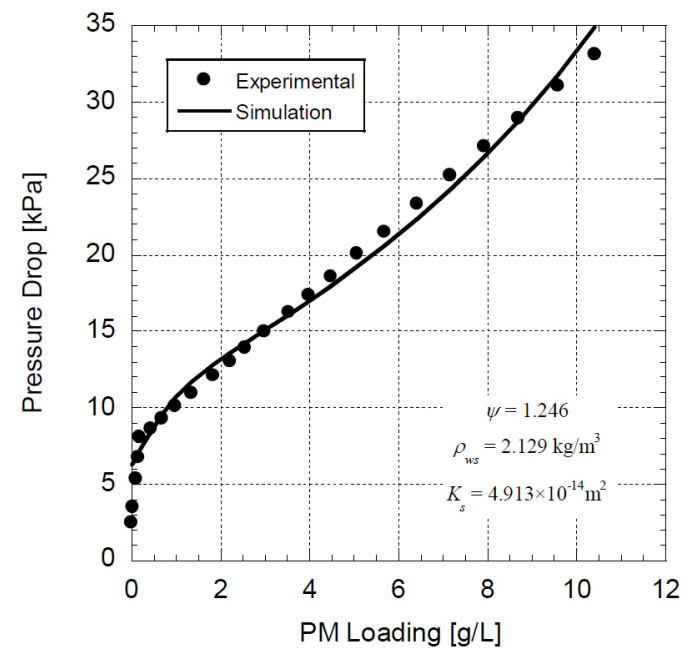

Figure 3. Calibration of PM loading parameters to the Young et al. experimental data [17].

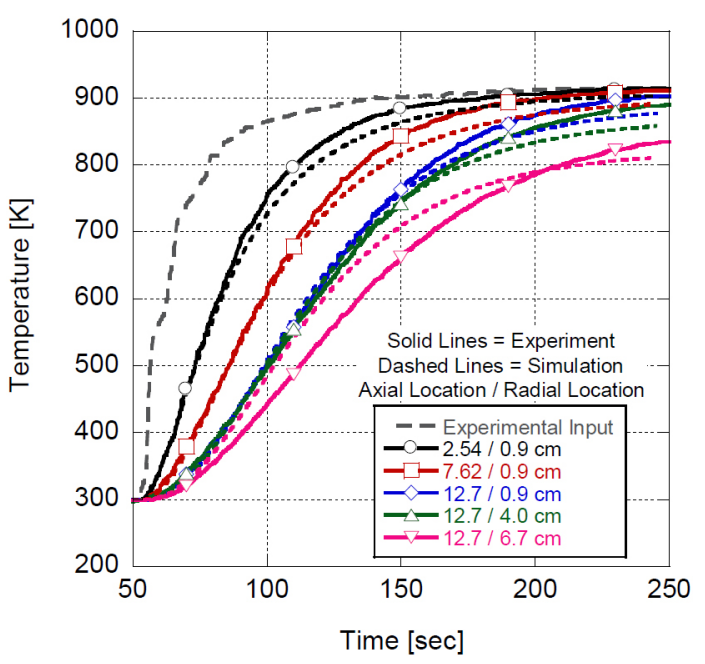

Figure 4. Comparison of Young et al. [17] experimental data and the mixed model running three axial zones and five radial zones after optimization.

In order to gauge the effectiveness of the deep bed filtration addition, the same loading simulation test accomplished prior with the two-channel model was utilized [12]. In the previous effort, only the cake layer was simulated using an area-conserved formulation in compressible flow. As illustrated by Figure 2, the mixed model presented here is able to capture the initial phases in the evolution of pressure drop. Investigating the calibrated parameters, the particulate 
permeability is different from the previous finding $\left(5.10 \times 10^{-14} \mathrm{~m}^{2}\right)$ with this deviation noted based on scheme differences. As for the particle density within the wall, the calibrated value found is significantly less than that of Konstandopoulos et al. [11]. As mentioned prior, this model does not include discretization in the wall direction, and lumps all PM capture into a single slab. Furthermore, it assumes that all pores in the wall are available for capturing PM, which is not true. Since it is not possible to measure this quantity, the value indicated is arbitrary and amounts to a physical curve-fit.

Similar to the previous effort, the experimental data of Young, et al. was simulated during both a warm-up experiment and oxidation event using three axial zones and five radial zones (total of 15 zones).

However, prior to simulating, the model was calibrated to the pressure drop data of Young, et al. in Figure 3. Then, the virtual filter was loaded with $37.11 \mathrm{gm}$ of PM as per the experimental test conditions. Of note, all parameters needed to run the simulation are provided in the earlier paper [ㄹ]

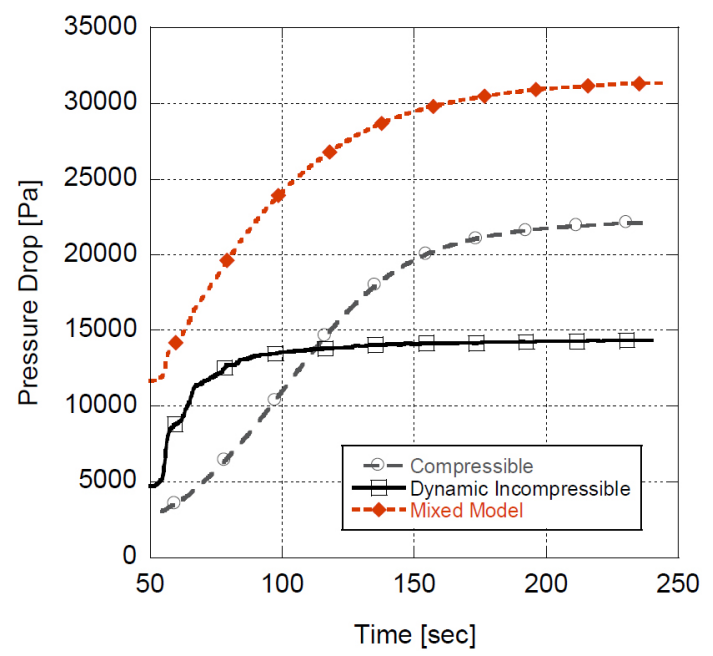

Figure 5. Comparison of the simulated pressure drop through the DPF using the two-channel (compressible) model and the lumped (incompressible) model.

Initially, when running the model it was found that the radial temperatures did not match when using the prior optimized (dynamic incompressibility model) estimates for the filter density, ambient convective heat transfer coefficient, and emissivity. However, because the run time averaged on the order of 363.60 seconds (not including the PM loading event for proper comparison with preceding efforts) with a standard deviation of 1.46 seconds* (previous model: $126.13 \pm 0.51$ seconds), it was still possible to optimize these variables to better match the results. This was accomplished using the fmincon optimization function in Matlab by minimizing the leastsquared deviation in temperature between the model and experimental data for all locations in Figure 4. The outcomes were a filter density of $1246.56 \mathrm{~kg} \mathrm{~m}^{-3}$, a convective coefficient of $32.03 \mathrm{~W}$ $\mathrm{m}^{-2} \mathrm{~K}^{-1}$ and an emissivity of 0.25 . As a result, Figure 4 ends up looking the same as the previous lumped model. This is anticipated because, as mentioned in an earlier section, there is only one term in the energy equations that is influenced by compressible flow; i.e., density in Eqn. (2). Since this term represents the storage of energy in the gas, it is relatively small as compared to the energy stored in the filter. Therefore, compressible flow does not significantly influence the warm-up of the filter.

However, compressible flow dramatically changes the pressure drop through the filter as indicated in Figure 5. When there is a relatively large pressure drop or temperature excursion, it is erroneous to apply the assumption of incompressibility even when the flow can be considered dynamically incompressible $(M \ll 1)$. Dynamic incompressibility removes the ideal gas law from the solution of the equations breaking the link between pressure and temperature. Incorporating a mixed model simulating an effective channel via compressible flow is able to recapture this connection and model the pressure drop similar to the classical two-channel approach including the time scale of change and the magnitude of the pressure drop. The difference between the compressible flow models is now a function of a more accurate pressure drop model via Figure 3.

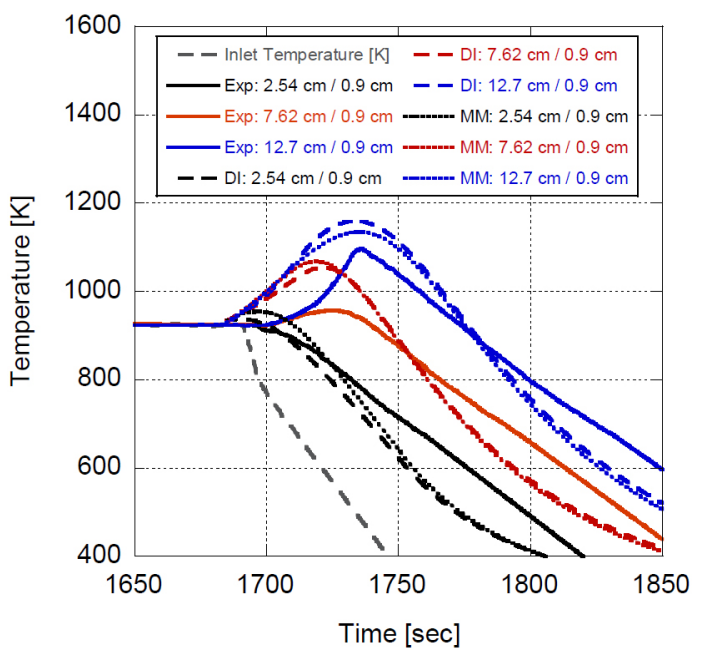

Figure 6. Comparison Young et al. [17] cool down and PM oxidation test with the dynamically incompressible (DI) and mixed model (MM) after calibration of the amount of PM converted.

When comparing the models during the Young et al. oxidation experiments via Figure 6 after calibration of the loading and PM oxidation amount (55\% converted), the temperature profiles are found to be similar. A greater deviation was noted than the warm up experiment because of a larger temperature difference across the DPF subsequently influencing the gas density. However, this change is not big enough to warrant the choice of the mixed model if temperature evolution is the only desired simulation outcome especially considering that the mixed model averaged 314.60 seconds with a standard deviation of 1.20 seconds; an increase of three fold in the numerical run time over the previous model (101.65 \pm 0.40 seconds). However, since Matlab runs approximately $10 \times$ slower than native programming languages, it is still possible to create a faster than real time model employing the combined approach in this paper (simulated run time was 199 seconds, with the $\mathrm{x}$-axis adjusted in order to match the Young et al. plots). 

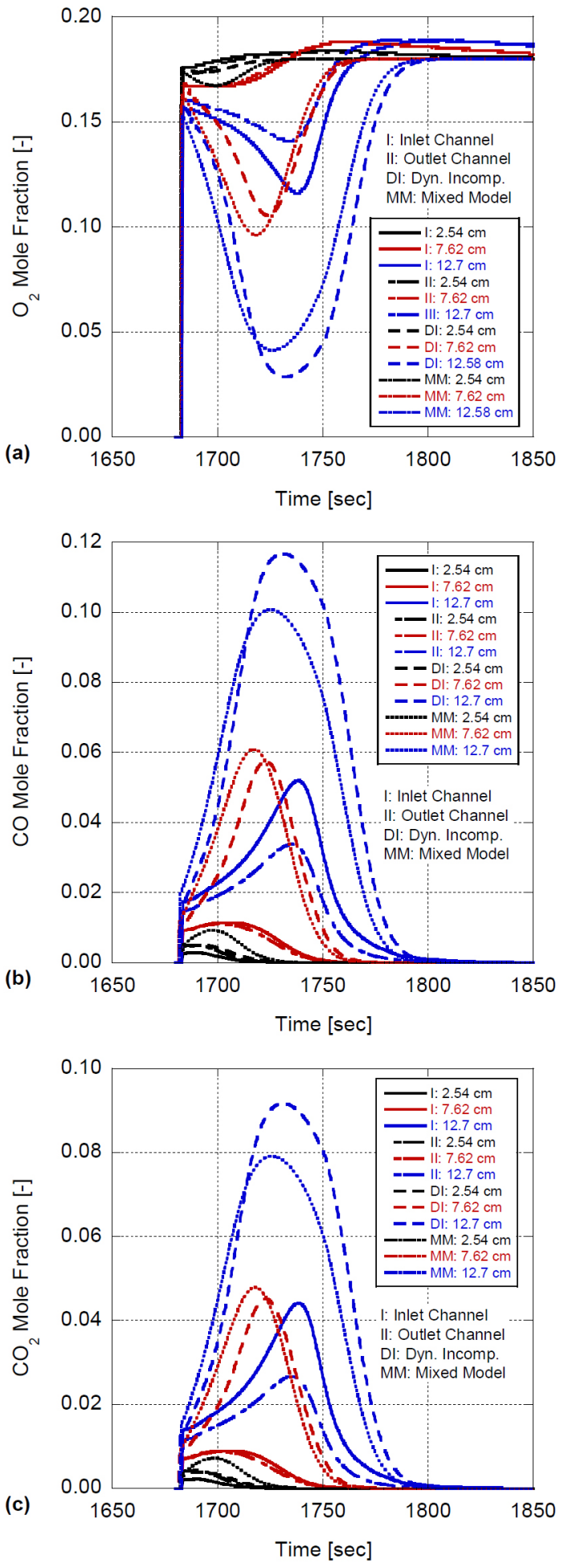

Figure 7. Mole fraction of (a) $\mathrm{O}_{2}$, (b) $\mathrm{CO}$, and (c) $\mathrm{CO}_{2}$ for the classical two-channel model (I and II) compared to the dynamically incompressible (DI) and mixed (MM) models.

Initially, it was thought that employing compressible flow would improve the prediction of the chemical species via Figure 7. However, as this figure elucidates, the mixed model does not appreciably help their accuracy. While the density change is now factored into the calculation of the concentrations in Eqn. (44), lumping chemical species into a single term cannot capture the complexity of mass transfer to and from the wall and the balance between diffusion and reactions occurring within the wall. Therefore, if chemical species are a desired outcome, they should be included as part of the Channel Equations. However, similar to the warm-up outcomes, the mixed model is now able to capture the interdependency of temperature and pressure as shown in Figure 8.

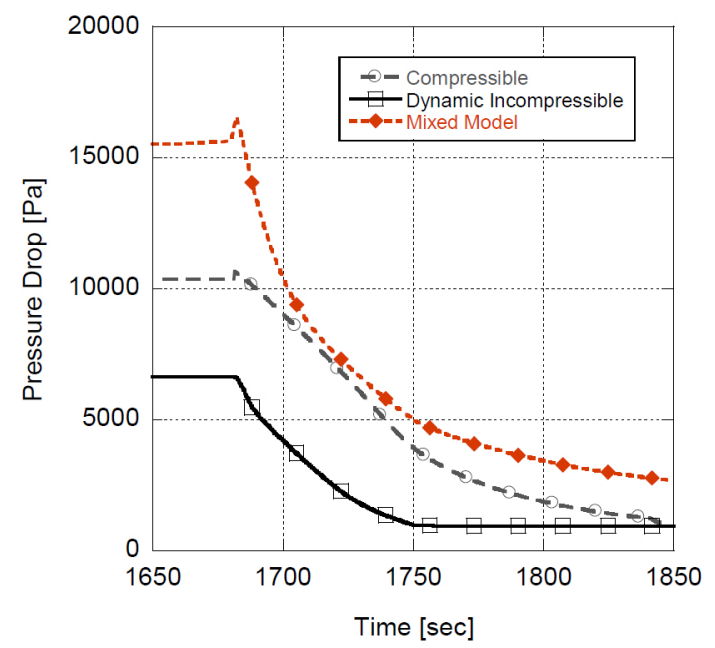

Figure 8. Pressure drop for the classical two-channel model compared to the dynamically incompressible and mixed models.

\section{CONCLUSIONS}

In a previous effort, a simplified particulate filter model was created that simulated the axial and radial zones of a filter using a resistance node network. Because of the favorable predicted outcome of temperature evolution, but erroneous pressure drop estimation, this model was combined with the classical two-channel approach in this paper. In specific, the assumption of dynamic incompressibility for the fluid flow regime was replaced with compressible flow effectively linking pressure and temperature through the ideal gas law. The results demonstrate that, with the addition of deep bed filtration, pressure drop can now be predicted relatively accurately along with the temperature. Furthermore, while the run time increased by approximately $3 x$, moving to a native programming language may still generate a faster than real time numerical tool. However, the estimation of chemical species still deviates significantly from the classical two channel approach. As a result, the following conclusions are noted:

- If a fast code is desired for implementation in an ECU and only temperature prediction is required, the resistance node model employing dynamic incompressibility should be utilized.

- If pressure drop prediction is also deemed necessary, then the mixed model presented in this effort provides a reasonable compromise of accuracy and numerical efficiency.

- If the estimation of chemical species is also wanted, then one should consider employing the classical two-channel modelling approach.

Future efforts involve simulating representative chemical species equations along with the mass and momentum equations while still employing the lumped model for temperature. This will conclude the analysis indicating whether or not it is possible to simulate chemical species differently than the full two-channel approach. 


\section{REFERENCES}

1. Diaz-Sanchez, D., "The Role of Diesel Exhaust Particles and their Associated Polyaromatic Hydrocarbons in the Induction of Allergic Airway Disease," Allergy, 52 (38 Suppl):52-6; discussion 57-8, 1997, doi:10.1111/j.1398-9995.1997.tb04871.x.

2. Depcik, C., Langness, C., and Mattson, J., "Development of a Simplified Diesel Particulate Filter Model Intended for an Engine Control Unit," SAE Technical Paper 2014-01-1559, 2014, doi:10.4271/2014-01-1559.

3. Koltsakis, G., Haralampous, O., Depcik, C., and Ragone, J. C., "Catalyzed Diesel Particulate Filter Modeling," Reviews in Chemical Engineering, 29 (1): 1-61, 2013, doi:10.1515/revce-2012-0008.

4. Bissett, E. J., "Mathematical Model of the Thermal Regeneration of a Wall-flow Monolith Diesel Particulate Filter," Chemical Engineering Science, 39 (7-8):1232-1244, 1984, doi:10.1016/0009-2509(84)85084-8.

5. Depcik, C., and Assanis, D., "Graphical User Interfaces in an Engineering Educational Environment," Computer Applications in Engineering Education, 13 (1):48-59, 2005, doi:10.1002/cae.20029.

6. Konstandopoulos, A. and Johnson, J., "Wall-Flow Diesel Particulate Filters-Their Pressure Drop and Collection Efficiency," SAE Technical Paper $\underline{\text { 890405, }}$, 1989, doi: $10.4271 / 890405$.

7. Konstandopoulos, A., Kostoglou, M., Skaperdas, E., Papaioannou, E. et al., "Fundamental Studies of Diesel Particulate Filters: Transient Loading, Regeneration and Aging," SAE Technical Paper 2000-01-1016, 2000, doi:10.4271/2000-01-1016.

8. Konstandopoulos, A., Skaperdas, E., and Masoudi, M., "Microstructural Properties of Soot Deposits in Diesel Particulate Traps," SAE Technical Paper 2002-01-1015, 2002, doi:10.4271/2002-01-1015.

9. Konstandopoulos, A., Kostoglou, M., Vlachos, N., and Kladopoulou, E., "Progress in Diesel Particulate Filter Simulation," SAE Technical Paper 2005-01-0946, 2005, doi:10.4271/2005-01-0946.

10. Fukushima, S., Ohno, K., Vlachos, N., and Konstandopoulos, A., "New Approach for Pore Structure and Filtration Efficiency Characterization," SAE Technical Paper 2007-01-1918, 2007, doi:10.4271/2007-01-1918.

11. Konstandopoulos, A., Kladopoulou, E., and Skaperdas, E., "Transient Pressure Drop of Diesel Particulate Filters," J. Aerosol Sci., 31 (Suppl. 1):S208-S209, 2000, doi:10.1016/S0021-8502(00)90215-5.

12. Depcik, C., and Assanis, D., "Simulating Area Conservation and the Gas-Wall Interface for One- Dimensional Based Diesel Particulate Filter Models," ASME Journal of Engineering for Gas Turbines and Power, 130 (6):062807, 2008, doi:10.1115/1.2939002.

13. Depcik, C., "Simulating the Concentration Equations and the Gas-Wall Interface for One-Dimensional Based Diesel Particulate Filter Models," ASME Journal of Engineering for Gas Turbines and Power, 132 (3):032803, 2010, doi:10.1115/1.3155792.

14. Haralampous, O., Koltsakis, G., and Samaras, Z., "Partial Regenerations in Diesel Particulate Filters," SAE Technical Paper 2003-01-1881, 2003, doi:10.4271/2003-01-1881.

15. Sprouse, C.III, Mangus, M., and Depcik, C., "Diesel Particulate FIlter Model with Detailed Permeability Analysis," ASME 2011 Internationa Mechanical Engineering Congress \& Exposition, Denver, CO, 2011, doi:10.1115/IMECE2011-63687.

16. Gantawar, A., Opris, C., and Johnson, J., "A Study of the Regeneration Characteristics of Silicon Carbide and Cordierite Diesel Particulate Filters Using a Copper Fuel Additive," SAE Technical Paper 970187 , 1997, doi:10.4271/970187.

17. Young, D., Warren, C., Gadkaree, K., and Johannesen, L., "Silicon Carbide for Diesel Particulate Filter Applications:Material Development and Thermal Design," SAE Technical Paper 2002-01-0324, 2002, doi:10.4271/2002-01-0324.

\section{CONTACT INFORMATION}

Christopher Depcik

Associate Professor

Department of Mechanical Engineering

depcik@ku.edu

$\mathrm{Ph}: 785-864-4151$

3144C Learned Hall

$1530 \mathrm{~W} .15^{\text {th }}$ Street

Lawrence

KS

66045-4709

\section{NOMENCLATURE}

$b \quad \underline{\text { Unit cell diameter }}$

$\bar{C} \quad$ Molar species concentration

$[\mathrm{m}]$

$c_{f} \quad$ Specific heat of filter material

$\left[\mathrm{mol} \mathrm{m}^{-3}\right]$

$\left[\mathrm{J} \mathrm{kg}^{-1}\right.$

$\left.\underline{\mathrm{K}}^{-1}\right]$

$c_{p} \quad$ Constant pressure specific heat

$\left[\mathrm{J} \mathrm{kg}^{-1}\right.$

$\left.\underline{\mathrm{K}}^{-1}\right]$

$c_{s} \quad \underline{\text { Specific heat of PM }}$

$\left[\mathrm{J} \mathrm{kg}^{-1}\right.$.

$\left.\underline{\mathrm{K}}^{-1}\right]$

$d \quad$ Side length of square channels $\quad$ [m]

$d c \quad$ Characteristic dimension of a unit cell collector [m]

$d c_{0} \quad$ Unloaded wall characteristic dimension of a unit [m] cell collector

$d c t \quad$ Average unit spherical collector in zone under study

$d p \quad$ Change in pressure during Newton-Raphson iteration

$d_{\text {pore }}$ Pore diameter

$F \quad$ Friction factor of Bissett (28.454)

$G \quad$ Flow variable

$G_{I I} \quad$ Outlet channel flow variable

$G_{\alpha} \quad \underline{\text { Variable transformed flow for inlet channel }}$

$k \quad$ Rate constant

$L_{t} \quad$ Total length of DPF

$\underline{\Delta L} \quad$ Effective zone length

$\dot{m} \quad$ Mass flow rate

$m s \quad$ Mass of PM in cake layer in each zone

$\dot{m} s \quad$ Mass flow rate of PM to the wall

mst Total PM stored in cake layer (radial zones)

$m w \quad$ Mass of PM in wall in each zone

$m w p \quad$ Mass of PM per pore

$\overline{m w p}$ Average wall PM mass per pore

$m w t \quad$ Total PM stored in wall (radial zones)

$\dot{m}_{\text {total }}$ Total mass flow rate into DPF

$[\mathrm{m}]$

$[\mathrm{Pa}]$

[m]

$[-]$

$\mathrm{kg} \mathrm{m}^{-2}-$

$\left.\underline{\mathrm{s}}^{-1}\right]$

$\left[\mathrm{kg} \mathrm{m}^{-2}\right.$ -

$\left.\underline{\mathrm{s}}^{-1}\right]$

$\mathrm{kg} \mathrm{m}^{-2}$

$\left.\underline{\mathrm{s}}^{-1}\right]$

$\left[\mathrm{m} \mathrm{s}^{-1}\right]$

[m]

[m]

$\left[\mathrm{kg} \mathrm{s}^{-1}\right]$

[kg]

$\left[\mathrm{kg} \mathrm{s}^{-1}\right]$

[kg]

$[\mathrm{kg}]$

$[\mathrm{kg}]$

[kg]

[kg]

$\left[\mathrm{kg} \mathrm{s}^{-1}\right]$

Nc Number of cells in each zone

[-]

$[-]$

$N c_{t} \quad$ Total number of cells

$\mathrm{Np} \quad$ Total number of pores at current location

Npa Total number of pores in the filter

Npt Total number of pores in the zone

$\mathrm{Nz} \quad$ Number of axial boundaries

$p_{I} \quad \underline{\text { Inlet channel pressure }}$

$p_{I I} \quad$ Outlet channel pressure

$p_{\text {amb }} \quad$ Ambient pressure

$p_{m} \quad$ Pressure at the interface between the PM and wall layers

$\dot{Q}_{\text {cond.axial }}$ Axial conduction

$\dot{Q}_{\text {cond, radial }}$ Radial conduction

$\dot{Q}_{\text {conv }} \quad$ Convection between channel gases and filter wall

$\dot{Q}_{\text {in }} \quad$ Enthalpy inlet flow to zone 
$\dot{Q}_{\text {out }} \quad$ Enthalpy outlet flow from zone

$\dot{Q}_{\text {reac }} \quad \underline{\text { PM exothermic reactions }}$

$\dot{Q}_{\text {wall }} \quad$ Enthalpy flow through wall

$R \quad$ Gas constant

$\overline{R R} \quad$ Species molar reaction rate

$S \quad$ Inlet channel surface area

Time

$T \quad$ Average gas temperature of channels

$\bar{T} \quad$ Weighted gas temperature average in the radial direction

$T_{\text {inlet }} \underline{\text { Inlet gas temperature }}$

$T_{I I I} \quad$ Average gas temperature of the channels at the $\underline{\text { boundaries }}$

Tf Temperature of combined filter and PM

ts $\quad$ PM thickness

$\overline{t s} \quad$ Average PM thickness across radial zones

tw Wall thickness

$u \quad$ Representative velocity in each zone

$u_{I} \quad$ Average inlet channel velocity

$u_{I I} \quad$ Average outlet channel velocity

$u_{\alpha} \quad$ Transformed velocity variable for inlet channel

$u_{s} \quad$ Velocity through PM layer

$\overline{u_{s}} \quad$ Average velocity through PM layer

$u_{w} \quad$ Velocity through wall layer

Veo Empty volume of the wall at the location under analysis

Ves Empty volume accounting for soot

$V f \quad$ Volume of filter in each zone

$V f t \quad$ Total solid filter volume

VS $\quad$ PM volume in each zone

$W \quad$ Molecular weight

$Y_{d} \quad$ Mass fraction of PM in inlet channel gas

$z \quad$ Axial distance
[W]

[W]

[W]

$\left[\mathrm{J} \mathrm{kg}^{-1}\right.$

$\left.\underline{\mathrm{K}}^{-1}\right]$

$\left[\mathrm{mol} \mathrm{m}^{-3}\right.$.

$\left.\underline{\mathrm{s}}^{-1}\right]$

$\left[\mathrm{m}^{2}\right]$

[s]

[K]

[K]

[K]

[K]

[K]

[m]

[m]

[m]

$\left[\mathrm{m} \mathrm{s}^{-1}\right]$

$\left[\mathrm{m} \mathrm{s}^{-1}\right]$

$\left[\mathrm{m} \mathrm{s}^{-1}\right]$

$\left[\mathrm{m} \mathrm{s}^{-1}\right]$

$\left[\mathrm{m} \mathrm{s}^{-1}\right]$

$\left[\mathrm{m} \mathrm{s}^{-1}\right]$

$\left[\mathrm{m} \mathrm{s}^{-1}\right]$

$\left[\mathrm{m}^{3}\right]$

$\left[\mathrm{m}^{3}\right]$

$\left[\mathrm{m}^{3}\right]$

$\left[\mathrm{m}^{3}\right]$

$\left[\mathrm{m}^{3}\right]$

$\left[\mathrm{kg} \mathrm{mol}^{-1}\right]$

[-]

[m]

\section{Greek Variables}

$\alpha_{\mathrm{O} 2} \quad \underline{\mathrm{O}}_{2}$ combustion partial factor $\quad[-]$

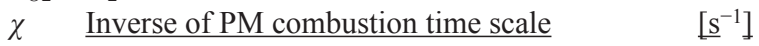

$\varepsilon \quad$ Porosity of loaded filter wall

$\varepsilon_{0} \quad$ Porosity of clean filter wall

$\underline{\text { Partition coefficient }}$

$K_{s} \quad$ Darcy's law permeability for PM layer

$[-]$

$K_{w} \quad$ Darcy's law permeability for wall layer

$\left[\mathrm{m}^{2}\right]$

$K_{w 0} \quad$ Darcy's law permeability for clean filter wall

$\mu \quad$ Dynamic viscosity

$\bar{\rho} \quad$ Average gas density of channels

$\left[\mathrm{m}^{2}\right]$

$\left[\mathrm{m}^{2}\right]$

$\left[\mathrm{kg} \mathrm{m}^{-1} \underline{\mathrm{s}}^{-1}\right]$

$\left[\mathrm{kg} \mathrm{m}^{-3}\right]$

$\rho_{I} \quad$ Gas density in inlet channel $\quad\left[\mathrm{kg} \mathrm{m}^{-3}\right]$

$\rho_{I I} \quad$ Gas density in outlet channel $\quad\left[\mathrm{kg} \mathrm{m}^{-3}\right]$

$\rho_{f} \quad$ Filter density

$\rho_{l} \quad$ Gas density in PM layer

$\left[\mathrm{kg} \mathrm{m}^{-3}\right]$

$\left[\mathrm{kg} \mathrm{m}^{-3}\right]$

$\rho_{s} \quad \underline{\text { PM density }}$

$\rho_{s w} \quad$ PM density in wall layer (calibrated)

$\left[\mathrm{kg} \mathrm{m}^{-3}\right]$

$\left[\mathrm{kg} \mathrm{m}^{-3}\right]$

$\rho_{w} \quad$ Gas density in wall layer $\quad\left[\mathrm{kg} \mathrm{m}^{-3}\right]$

$\psi \quad$ Percolation factor (constant \& calibrated) $\quad[-]$

\section{SUBSCRIPTS AND SUPERSCRIPTS}

$\boldsymbol{i}$ - Radial direction

$\boldsymbol{j}$ - Axial direction

$\boldsymbol{m}$ - Boundaries

' - First iterative result

" - Second iterative result

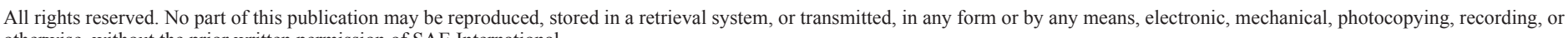
otherwise, without the prior written permission of SAE International. 